\title{
20. GEOCHEMISTRY OF THE CRETACEOUS/TERTIARY BOUNDARY AT HOLE 752B, BROKEN RIDGE ${ }^{1}$
}

\author{
Robert M. Owen ${ }^{2}$ and Andrew R. B. Zimmerman ${ }^{2}$
}

\begin{abstract}
Statistical analyses of sediment geochemical data from a Cretaceous/Tertiary (KT) boundary section at Broken Ridge indicate that the sediments here are composed of three major geochemical end-members: (1) a biogenic/dissolution residue component; (2) a volcanogenic component; and (3) a diagenetic/alteration product component. The KT boundary at Broken Ridge is overlain by a 6-m-thick ash-rich layer. Relative flux comparisons of the three geochemical end-members suggest, however, this layer is the result of a dramatic (11-fold) decrease in biological productivity immediately following the KT boundary event, while both the flux and composition of the volcanogenic component remain constant across the KT boundary. The volcanogenic component apparently is derived from a local source, and we find no evidence of a causal link between volcanic activity and the KT boundary event. The flux of the diagenetic component, which is enriched in Au, increases sharply at the KT boundary. All other elements associated with this component are present in significant amounts in the other two geochemical end-members. With the possible exception of Au, then, geochemical anomalies in the sediments just below the KT boundary appear to result from diagenetic alteration of biogenic and volcanogenic sediment precursors.
\end{abstract}

\section{INTRODUCTION}

An unexpected accomplishment of drilling at Broken Ridge was the recovery of an expanded section of sediment across the Cretaceous/Tertiary boundary (KT boundary) at Hole 752B. We have conducted a geochemical analysis of this sedimentary section to obtain information relevant to the ongoing discussion concerning the probable causes and ultimate effects of globalscale processes which occurred during this period of earth history.

Analyses of KT boundary sediments from many locations are characterized by geochemical anomalies involving enrichments in Ir and other noble metals ( $\mathrm{Au}, \mathrm{Pd}, \mathrm{Pt}$ ), as well as certain siderophile $(\mathrm{Cr}, \mathrm{Co}, \mathrm{Ni})$ and chalcophile (As, $\mathrm{Sb}, \mathrm{Zn}, \mathrm{Cu}, \mathrm{Se}$ ) elements (Alvarez et al., 1980; Kyte et al., 1980; Ganapathy, 1980; Gilmore et al., 1984; Smit and ten Kate, 1982; Schmitz et al., 1988), and depletions in rare earth element (REE) concentrations (Smit and ten Kate, 1982; DePaolo et al., 1983). At present no consensus exists concerning the significance of these geochemical anomalies, although two major hypotheses have been proposed. Some authors (Alvarez et al., 1980, 1982) have suggested that the positive Ir anomalies may reflect the impact of a large meteorite upon the earth, while others (McLean, 1985; Officer and Drake, 1983; Officer et al., 1987) have argued that the Ir anomalies and enrichments of $\mathrm{Se}, \mathrm{Sb}$, and As in KT boundary sediments could have resulted from a period of widespread volcanism. The resolution of this debate is complicated by the fact that the observed geochemical anomalies involve both meteoritic and non-meteoritic elements, the meteoritic elements do not point to a single known meteorite composition (Kyte et al., 1980; Kyte and Wasson, 1982; Crocket et al., 1988), and the possibility that the sediments may have been "contaminated" by ejecta material (Kastner et al., 1984) or altered by diagenetic effects (Schmitz, 1985; Schmitz et al., 1988; Dyer et al., 1989).

\footnotetext{
'Weissel, J., Peirce, J., Taylor, E., Alt, J., et al., 1991. Proc. ODP, Sci. Results, 121: College Station, TX (Ocean Drilling Program).

2 Department of Geological Sciences, The University of Michigan, Ann Arbor, MI 48109-1063, U.S.A.
}

Whatever the cause of the KT boundary event, there is general agreement that its effects were both pronounced and ubiquitous. Analyses of KT boundary sections from both Atlantic and Pacific DSDP cores reveal significant reductions in carbonate accumulation rates and a drastic reduction in the diversity of marine phytoplankton and other microfossil species (Tappan, 1968; Fischer and Arthur, 1977; Arthur et al., 1987). Considering the high degree of preservation of these calcareous microfossils, these changes can not, in many cases, be attributed simply to increased dissolution (Thierstein, 1981; Zachos and Arthur, 1986). Furthermore, negative perturbations in the $\delta^{13} \mathrm{C}$ of marine surface waters and surface to deepwater $\delta^{13} \mathrm{C}$ gradients are considered as evidence of a primary productivity minimum which may have lasted a million years following the boundary event (Zachos and Arthur, 1986).

The recovery of a KT boundary section during ODP Leg 121 provides an opportunity to add to our knowledge about this period by developing information from the Indian Ocean and from an austral/temperate setting. The sample suite examined here represents the time interval between 67.5 and $64.2 \mathrm{Ma}$, and thus spans the KT boundary (66.4 Ma, Peirce, Weissel, et al., 1989). We note that these analyses cannot be construed as a high resolution study of a complete sedimentary section because the boundary sediments were recovered in the form of "drilling biscuits" (see following discussion) and it is likely that some sedimentary material is missing. Consequently, the primary aim of this study has been to highlight similarities and/or differences, and to delineate significant changes in sediment geochemistry which occurred during the period represented by the onset, occurrence, and recovery from the KT boundary event at Broken Ridge. Our analyses include many of those elements which are significant to the debate concerning the possible cause(s) of the mass extinctions at the KT boundary, as well as those which can serve as proxy indicators of the effects of the biological productivity crisis.

\section{LITHOLOGIC SETTING}

The KT boundary was recovered at Hole 752B in Core 121$752 \mathrm{~B}-11 \mathrm{R}$ ( $52 \%$ recovery) at about $358 \mathrm{~m}$ below the seafloor (mbsf). Preliminary shipboard analyses indicate a major decrease in Cretaceous calcareous microfossil diversity at $358.75 \mathrm{mbsf}$ and 
the first appearance of Tertiary nannofossils at $358.53 \mathrm{mbsf}$ (Peirce, Weissel, et al., 1989). Based on global lithologic correlation, the location of the KT boundary is assumed to occur within the interval between 358.7 and $358.75 \mathrm{mbsf}$ (Peirce, Weissel, et al., 1989) in this core. The boundary occurs within an ash-chertchalk sequence which is underlain by light grey Cretaceous chalks and overlain by $6 \mathrm{~m}$ of dark green, ash-rich sediment (see Peirce, Weissel, et al., 1989 for a stratigraphic section diagram). This thick ash layer is, in turn, overlain by Paleocene chalks. Fine laminations, burrows, and mottles are found in the ash and chalk layers of the $60 \mathrm{~cm}$ boundary section. Because the boundary section was recovered in the form of "drilling biscuits," we must keep in mind that some sediment, most likely that associated with chert layers, may be missing. The flora of the boundary section and the overlying ash-rich layer exhibit a sharp decrease in species diversity and contain an assemblage of opportunistic "survivor" species (Peirce, Weissel, et al., 1989). The lithostratigraphy of this KT boundary section is atypical of other studied boundary sections only with respect to the voluminous ash deposits with which it is associated. Shipboard estimates of sediment flux across the boundary suggest the ash-rich layer is primarily a reflection of reduced carbonate flux rather than a sudden sharp influx of volcanic debris (Rea et al., 1990). An increase in Tertiary nannofossil diversity occurs at $354.5 \mathrm{mbsf}$, about $66 \mathrm{Ma}$ (Peirce, Weissel, et al., 1989) and is followed by a return to normal (i.e., Maestrichtian) productivity levels between 63.8 and 64.8 Ma (Rea et al., 1990).

\section{METHODS}

Geochemical analyses were performed on a suite of 44 samples taken from sediment deposited over 3.3 m.y. (64.2-67.5 Ma) ranging from the upper Maastrichtian, through the KT boundary and the $6 \mathrm{~m}$ ash-rich layer, and ending in the lower Paleocene. Except as noted below, samples were collected at $1.5 \mathrm{~m}$ intervals from $30 \mathrm{~m}$ above to $27 \mathrm{~m}$ below the KT boundary (328-385 mbsf). A higher resolution suite of 24 samples was collected from the interval between 66 and $66.4 \mathrm{Ma}$. This suite corresponds to sampling intervals of $10 \mathrm{~cm}$ and $30 \mathrm{~cm}$, respectively, for the boundary layer sediments (Section 121-752B-11R-3), and for the two overlying core sections. We emphasize that our analyses do not include material from the presumed KT boundary layer (121752B-11R-3, 90-5 cm); analyses of this layer are discussed elsewhere in this volume. All samples, consisting of 1-2 cm sections of core, were weighed, freeze-dried, and reweighed so that dry bulk density could be calculated. Samples were then ground with mortar and pestle and homogenized. Carbonate content was determined using a conventional pressure bomb technique. All elemental abundances were determined by instrumental neutron activation analysis (INAA) at the Phoenix Memorial Laboratory, The University of Michigan, using standard INAA procedures (Gordon et al., 1968; Dams and Robbins, 1970). Replicate analyses of NBS-1633A standard as well as Standard Pacific Sediment (Owen and Ruhlin, 1986) were performed to evaluate analytical precision (Table 1).

The statistical methods employed in this study are designed to discriminate between different types of sediment in the samples and to determine the relative contribution of each type to any given sediment sample. Both the number and the chemical composition of the major geochemical end-members in our samples are determined by a quantitative sediment partitioning procedure, which involves a mathematical manipulation of the geochemical data set using multivariate Q-mode factor analysis and vector rotation according to the technique of Leinen and Pisias (1984). The chemical significance (i.e., the nature of the source material) of each end-member identified by this procedure is determined by comparing key inter-element ratios within each end-member with analogous ratios reported in the literature and known to be characteristic of pure geochemical source materials (Dymond, 1981; Graybeal and Heath, 1984; Leinen and Pisias, 1984). The relative amount of each end-member in each sample is determined by a linear programming procedure which provides an optimum solution (minimum error) for each sample. The computer programs needed to implement this technique were kindly provided to us by Drs. Leinen and Pisias.

The biostratigraphy used here is based on the nannofossil stratigraphy as determined on board ship and as slightly revised at the Leg 121 post-cruise meeting in January of 1989 (Peirce, Weissel, et al., 1989). For Cenozoic materials, we have used the zonations of Okada and Bukry (1980) as tied to the time scale of Berggren et al. (1985). Mesozoic stratigraphy is based on the zonation of Sissingh (1977) as tied to the magnetic reversal time scale of Harland et al. (1982) using the revisions proposed by Kent and Gradstein (1985). The total sediment mass accumulation rate (MAR) for each sample (Table 2) is a quantification of the true mass flux of sedimentary material to the seafloor, and is calculated as the product of the dry bulk density and the linear sedimentation rate. When the total MAR value for each sample is multiplied by the weight percent of any sediment component in that sample, the flux of that component is also quantified, and so the pitfalls of interpreting relative abundance data can be avoided. Dry bulk density (DBD) for each sample was determined from our porosity measurements, where $\mathrm{DBD}=2.65$ (100-\% porosity). Linear sedimentation rates are based on the nannofossil stratigraphy. A summary of the age-depth-LSR values for the core intervals discussed here are reported in Rea et al. (1990) and, for convenience, are also shown here in Table 3 . We note that because of the limitations of LSR data, which are determinable for every nannofossil zone, interpretations of the resulting MAR values are useful for deciphering phenomena that occur on tectonic time scales. Rea et al. (1990) have estimated the accuracy of the MAR data to be $\pm 20 \%$; minor changes may not be significant.

\section{RESULTS AND DISCUSSION}

\section{Compositional End-members}

The downcore distributions of 28 elemental concentrations (calculated on a carbonate-free basis), $\mathrm{CaCO}_{3}$ content, and whole sediment MAR's are listed in Table 2. Several preliminary statistical analyses were performed to identify groups of elements which exhibit a coherent chemical behavior. Where appropriate, a single representative element (e.g., $\mathrm{Ba}$, for $\mathrm{Ba}, \mathrm{Sr}$, and $\mathrm{Br}$ ) was used in the final factor analysis to avoid significant redundancy in the data set.

The factor analysis identified three factors which collectively account for $91.8 \%$ of the total variance in the data (Table 4). We interpret Factor 1 as representing biogenic material and the dissolution residue of this material. Several inter-elemental ratios in this factor are typical of those reported in the literature for marine plankton $(\mathrm{Mn} / \mathrm{Ba}, \mathrm{Al} / \mathrm{Ba}, \mathrm{Co} / \mathrm{Zn}, \mathrm{Al} / \mathrm{Mn}$; Martin and Knauer, 1973; Dymond, 1981; Graybeal and Heath, 1984; Palmer, 1985), foraminifera tests $(\mathrm{La} / \mathrm{Yb}, \mathrm{La} / \mathrm{Sm}, \mathrm{Sm} / \mathrm{Eu} ;$ Palmer, 1985) and/or for the dissolution residue of carbonate and siliceous organisms (Zn/Al, Mn/Al; Dymond, 1981; Graybeal and Heath, 1984). Barium, which is believed to be strongly associated with biogenic material (Gurvich et al., 1978; Dehairs et al., 1980) and can be used as a proxy for enhanced biological productivity (Boström et al., 1973; Schmitz, 1987), is most heavily loaded in Factor 1. Manganese, also loaded in Factor 1, has been found to be enriched in phytoplankton (Riley and Roth, 1971; Martin and Knauer, 1973). In preliminary statistical runs, $\mathrm{Sr}$, which known to substitute for $\mathrm{Ca}$ in carbonates, and $\mathrm{Br}$, an element associated with organic carbon (Price and Calvert, 1977; Mayer et al., 1981) were 
Table 1. Summary of replicate analyses of Pacific Mud and NBS-1633A Standard (data in ppm).

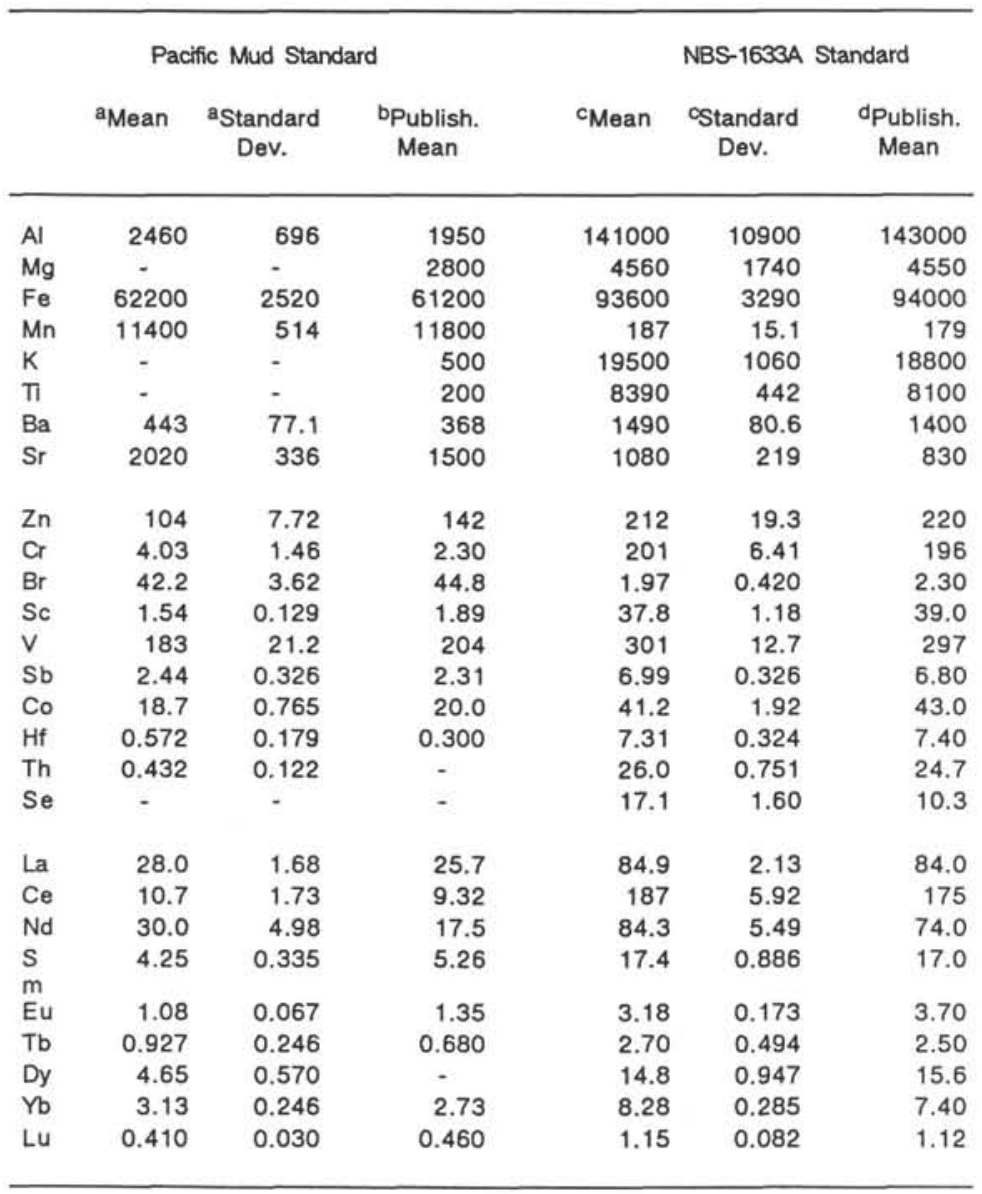

\footnotetext{
a U.M. INAA, based upon 17 analyses.

bOwen and Ruhlin (1986).

c U. M. INAA, based upon 13 analyses.

d Gladney et al. (1987).
}

also strongly associated with this factor. Consequently, we have used downcore variations in Factor 1 as a proxy indicator of paleoproductivity trends.

Factor 2, which accounts for $36.7 \%$ of the variability in the data set, is interpreted as a volcanogenic sediment component. It is well-documented that there are significant variations in the composition of volcanogenic materials in marine sediments throughout the world ocean (e.g., Chester and Aston, 1976). Thus, while our interpretation of Factor 2 is consistent with the large amounts of ash present in this core section, it cannot be evaluated in terms of any universally accepted set of geochemical criteria for identifying volcanogenic materials, because no such criteria exist. In this case, however, several inter-element ratios of Factor 2 do compare well (Table 5) with those of known volcanogenic materials from the same region, including southern Ninetyeast Ridge volcanogenic sediment (DSDP Site 253, Fleet et al., 1976; Fleet and McKelvey, 1978), a Santonian ash layer (ODP Hole 121-755A), and Kerguelen Plateau basalts (Storey et al., 1988).

The composition of Factor 3, which accounts for $12.1 \%$ of the total variance, is similar in some respects to that of both biogenic materials (Factor 1) and volcanogenic materials (Factor 2). Unlike Factors 1 or 2, however, Factor 3 is enriched in the noble metal
Au, although it has been suggested that noble metals may be enriched in both volcanic ash (Goldschmidt, 1954; Zoller et al., 1983; Olmez et al., 1986) and organic materials (Crocket and Kuo, 1979; Schmitz et al., 1988) through diagenetic processes. For these reasons, we interpret Factor 3 as reflecting a diagenetic alteration product of the biogenic and volcanogenic sediment represented by the first two end-members. Although both Factor 2 and 3 are enriched in $\mathrm{Fe}$, the $\mathrm{Mn} / \mathrm{Fe}$ ratios of these end-members appear to be too low to be indicative of the presence of any significant amounts of hydrothermal or authigenic metalliferous sedimentary components (Dymond, 1981; Graybeal and Heath, 1984). These sediments also do not display As enrichments (Marchig et al., 1982) or the very negative Ce anomalies and heavy REE enriched patterns typical of hydrothermal sediments (Piper, 1974a; Ruhlin and Owen, 1986), nor do they display the positive $\mathrm{Ce}$ anomalies found in authigenic ferromanganous sediments (Piper, 1974b; Elderfield et al., 1981).

The REE patterns of individual sediment samples (Fig. 1) are grossly similar. All show distinct light REE enrichment and absolute abundances similar to those of basalts from the Kerguelen Plateau and Ninetyeast Ridge (Fleet et al., 1976; Reddy et al., 1978), or more generally, from an ocean island or alkalic 
Table 2. Major and trace element data determined by INAA (data in ppm except where indicated), sample ages, whole sediment mass accumulation rates (MAR), and carbonate content. For statistical analysis, we assume elemental concentrations equal to one-half the detection limit where indicated by the $(<)$ symbol. The data presented here are averages of two analyses and are calculated on a carbonate-free basis.

\begin{tabular}{|c|c|c|c|c|c|c|c|c|c|c|}
\hline Sample & mbsf & $\begin{array}{l}\text { Age } \\
(\mathrm{Ma})\end{array}$ & $\begin{array}{c}\text { Al } \\
(\%)\end{array}$ & $\begin{array}{l}\mathrm{Mg} \\
(\%)\end{array}$ & $\begin{array}{l}\mathrm{Fe} \\
(\%)\end{array}$ & $\mathrm{Mn}$ & $\begin{array}{c}K \\
(\%)\end{array}$ & $\begin{array}{c}T i \\
(\%)\end{array}$ & $\begin{array}{l}\mathrm{Ba} \\
(\%)\end{array}$ & $\begin{array}{c}\mathrm{Sr} \\
(\%)\end{array}$ \\
\hline \multicolumn{11}{|l|}{ ODP Hole 121-752B } \\
\hline $8 \mathrm{R}-03,132-135 \mathrm{~cm}$ & 328.72 & 64.18 & 3.51 & 2.13 & 3.19 & 2940 & $<0.806$ & 0.530 & 0.742 & 0.619 \\
\hline $8 \mathrm{R}-04,132-135 \mathrm{~cm}$ & 330.22 & 64.24 & 1.82 & 0.865 & 1.81 & 1580 & $<0.437$ & $<0.132$ & 0.424 & 0.252 \\
\hline $8 R-05,131-134 \mathrm{~cm}$ & 331.71 & 64.29 & 4.50 & 3.57 & 6.48 & 1213 & 1.98 & 0.798 & 0.586 & 0.234 \\
\hline $8 \mathrm{R}-06,132-135 \mathrm{~cm}$ & 333.22 & 64.35 & 3.32 & 1.31 & 3.14 & 1500 & 2.53 & 0.398 & 0.639 & 0.263 \\
\hline $8 R-07,132-135 \mathrm{~cm}$ & 334.72 & 64.41 & 5.47 & 3.17 & 7.20 & 743 & 2.33 & 0.837 & 0.325 & 0.178 \\
\hline $9 R-01,129-132 \mathrm{~cm}$ & 336.69 & 64.48 & 2.21 & 1.30 & 2.31 & 1730 & 2.50 & 0.520 & 0.579 & 0.405 \\
\hline $10 \mathrm{R}-01,54-57 \mathrm{~cm}$ & 345.64 & 64.87 & 3.87 & 1.51 & 3.38 & 2051 & 1.78 & 0.634 & 0.603 & 0.720 \\
\hline $10 \mathrm{R}-02,54-57 \mathrm{~cm}$ & 347.14 & 65.06 & 6.08 & 3.24 & 7.09 & 1810 & 2.40 & 1.02 & 0.468 & 0.520 \\
\hline $10 \mathrm{R}-03,54-57 \mathrm{~cm}$ & 348.64 & 65.24 & 5.11 & 3.28 & 6.05 & 2200 & 0.847 & 0.639 & 0.515 & 0.494 \\
\hline $10 \mathrm{R}-04,53-56 \mathrm{~cm}$ & 350.13 & 65.43 & 3.92 & 2.79 & 6.50 & 2800 & 2.02 & 0.702 & 0.608 & 0.765 \\
\hline $10 \mathrm{R}-05,53-56 \mathrm{~cm}$ & 351.63 & 65.62 & 1.27 & 0.359 & 0.975 & 762 & 0.509 & 0.202 & 0.246 & 0.136 \\
\hline $10 \mathrm{R}-06,55-58 \mathrm{~cm}$ & 353.15 & 65.81 & 5.71 & 3.38 & 7.42 & 621 & 2.11 & 0.859 & 0.397 & 0.137 \\
\hline $11 \mathrm{R}-01,30-32 \mathrm{~cm}$ & 355.10 & 66.03 & 5.69 & 3.27 & 8.67 & 705 & 1.83 & 1.29 & 0.077 & 0.129 \\
\hline $11 \mathrm{R}-01,70-72 \mathrm{~cm}$ & 355.50 & 66.07 & 5.66 & 2.94 & 6.73 & 590 & 1.80 & 1.00 & 0.162 & 0.203 \\
\hline $11 \mathrm{R}-01,110-112 \mathrm{~cm}$ & 355.90 & 66.12 & 4.63 & 2.77 & 6.87 & 611 & 1.83 & 0.947 & 0.317 & 0.146 \\
\hline $11 \mathrm{R}-01,140-142 \mathrm{~cm}$ & 356.20 & 66.15 & 4.90 & 2.69 & 6.42 & 531 & 1.86 & 0.888 & 0.261 & 0.197 \\
\hline $11 \mathrm{R}-02,30-32 \mathrm{~cm}$ & 356.60 & 66.19 & 4.43 & 3.70 & 8.34 & 601 & 1.57 & 0.368 & 0.273 & 0.174 \\
\hline $11 \mathrm{R}-02,70-72 \mathrm{~cm}$ & 357.00 & 66.24 & 4.67 & 3.03 & 6.76 & 540 & 2.27 & 0.952 & 0.322 & 0.127 \\
\hline $11 \mathrm{R}-02,110-112 \mathrm{~cm}$ & 357.40 & 66.28 & 4.85 & 2.91 & 6.38 & 619 & 1.76 & 1.12 & 0.359 & 0.183 \\
\hline $11 \mathrm{R}-02,140-142 \mathrm{~cm}$ & 357.70 & 66.31 & 4.31 & 2.95 & 6.31 & 849 & 2.15 & 0.656 & 0.331 & 0.213 \\
\hline $11 \mathrm{R}-03,20-21 \mathrm{~cm}$ & 358.00 & 66.35 & 4.36 & 3.63 & 7.26 & 674 & 1.44 & 0.550 & 0.405 & 0.228 \\
\hline $11 \mathrm{R}-03,35-36 \mathrm{~cm}$ & 358.15 & 66.36 & 4.16 & 3.45 & 7.98 & 809 & 1.96 & 0.891 & 0.627 & 0.257 \\
\hline $11 \mathrm{R}-03,50-51 \mathrm{~cm}$ & 358.30 & 66.38 & 2.38 & 1.50 & 1.97 & 3550 & $<0.451$ & 0.329 & 0.592 & 0.188 \\
\hline $11 \mathrm{R}-03,65-66 \mathrm{~cm}$ & 358.45 & 66.39 & 4.87 & 4.55 & 8.30 & 1570 & 1.57 & 0.741 & 0.389 & 0.198 \\
\hline $11 \mathrm{R}-03,80-81 \mathrm{~cm}$ & 358.60 & 66.40 & 1.73 & 1.46 & 1.15 & 5170 & $<0.678$ & $<0.178$ & 0.370 & 0.281 \\
\hline $11 \mathrm{R}-03,97-98 \mathrm{~cm}$ & 358.77 & 66.41 & 2.19 & 1.27 & 2.57 & 2140 & 1.74 & 0.355 & 0.446 & 0.483 \\
\hline $11 \mathrm{R}-03,105-106 \mathrm{~cm}$ & 358.85 & 66.41 & 3.99 & 1.56 & 3.74 & 2340 & 2.48 & 0.715 & 0.447 & 0.614 \\
\hline $11 \mathrm{R}-03,115-116 \mathrm{~cm}$ & 358.95 & 66.42 & 2.91 & 2.15 & 2.29 & 2960 & 2.03 & 0.606 & 0.526 & 0.713 \\
\hline $11 \mathrm{R}-03,125-126 \mathrm{~cm}$ & 359.05 & 66.42 & 2.63 & 1.27 & 1.90 & 2770 & 1.92 & 0.693 & 0.525 & 0.657 \\
\hline $11 \mathrm{R}-03,135-136 \mathrm{~cm}$ & 359.15 & 66.43 & 2.81 & 1.20 & 1.75 & 2340 & 1.64 & 0.149 & 0.497 & 0.459 \\
\hline $12 \mathrm{R}-01,139-142 \mathrm{~cm}$ & 365.79 & 66.70 & 5.98 & 1.83 & 4.40 & 2160 & 3.51 & 0.783 & 0.564 & 0.767 \\
\hline $12 \mathrm{R}-02,136-139 \mathrm{~cm}$ & 367.26 & 66.76 & 6.02 & 3.98 & 8.29 & 2300 & 2.07 & 1.10 & 0.441 & 0.725 \\
\hline $12 \mathrm{R}-03,70-73 \mathrm{~cm}$ & 368.10 & 66.79 & 4.19 & 1.11 & 2.14 & 2570 & 2.04 & 0.715 & 0.804 & 0.836 \\
\hline $12 \mathrm{R}-04,68-71 \mathrm{~cm}$ & 369.58 & 66.85 & 4.73 & 2.15 & 3.90 & 3010 & 3.55 & 1.09 & 0.782 & 0.930 \\
\hline $12 \mathrm{R}-05,37-40 \mathrm{~cm}$ & 370.77 & 66.90 & 4.40 & 1.38 & 2.91 & 2280 & 2.42 & 0.346 & 0.629 & 0.688 \\
\hline $12 \mathrm{R}-06,48-51 \mathrm{~cm}$ & 372.38 & 66.97 & 4.35 & 1.95 & 5.33 & 1620 & 3.10 & 0.697 & 0.566 & 0.644 \\
\hline $13 \mathrm{R}-01,2-5 \mathrm{~cm}$ & 374.02 & 67.03 & 4.35 & 2.06 & 2.43 & 2340 & 2.60 & 0.570 & 0.682 & 0.779 \\
\hline $13 \mathrm{R}-02,51-54 \mathrm{~cm}$ & 376.01 & 67.11 & 7.14 & 2.57 & 4.82 & 1340 & 2.98 & 0.898 & 0.555 & 0.583 \\
\hline $13 \mathrm{R}-03,142-145 \mathrm{~cm}$ & 378.42 & 67.21 & 6.02 & 3.54 & 7.26 & 2620 & 2.79 & 0.704 & 0.555 & 0.884 \\
\hline $13 \mathrm{R}-04,43-46 \mathrm{~cm}$ & 378.93 & 67.23 & 5.40 & 4.93 & 6.30 & 2600 & 2.18 & 0.779 & 0.491 & 0.586 \\
\hline $13 R-05,91-94 \mathrm{~cm}$ & 380.91 & 67.31 & 5.95 & 2.92 & 8.16 & 676 & 2.70 & 0.768 & 0.351 & 0.332 \\
\hline $13 \mathrm{R}-06,47-50 \mathrm{~cm}$ & 381.97 & 67.36 & 7.00 & 2.32 & 6.31 & 1220 & 3.04 & 0.896 & 0.304 & 0.533 \\
\hline $14 \mathrm{R}-01,57-60 \mathrm{~cm}$ & 384.17 & 67.45 & 5.67 & 3.95 & 5.37 & 3600 & 2.82 & 1.12 & 0.761 & 1.16 \\
\hline $14 \mathrm{R}-02,48-51 \mathrm{~cm}$ & 385.58 & 67.50 & 7.36 & 3.13 & 4.01 & 5000 & $<1.53$ & $<0.487$ & 0.816 & 1.68 \\
\hline
\end{tabular}

basaltic source. Submarine weathering of basalt has been shown to produce light REE enrichment (Ludden and Thompson, 1978; Despraires and Bonnot-Courtois, 1980); however, the effect of diagenetic alteration on the REE's of volcanogenic sediment is relatively unknown (Fleet et al., 1976). Some of the REE patterns also display slightly negative $\mathrm{Ce}$ anomalies, which may be due to the presence of calcareous or siliceous biogenic sediments which are commonly Ce depleted (Piper, 1974a, b; Shimizu and Masuda, 1977; Palmer, 1985). The most negative Ce anomalies occur in those samples which best reflect the composition of Factor 1, and thus they support our interpretation of this factor being composed of biogenic material derived from seawater, which is characterized by a pronounced negative $\mathrm{Ce}$ anomaly (Fleet, 1984).

\section{End-member Distributions}

Downcore profiles of both the relative amount (Fig. 2A-C) and MAR (Fig. 3A-C) of each geochemical end-member are used here as an aid in interpreting the paleoceanography of the KT section. The profile of the biogenic end-member (Factor 1) closely follows that of carbonate content (Fig. 4). There is a drastic reduction in both the relative abundance (Fig. 2A) and MAR (Fig. 3A) of this end-member at $358.45 \mathrm{mbsf}(66.39 \mathrm{Ma})$ just above the KT boundary. We report an 11-fold reduction in the MAR of the biogenic component (from 3.3 to $0.3 \mathrm{~g} / \mathrm{cm}^{2} / \mathrm{k}$.y.) across the KT boundary. The MAR of the biogenic component does not return to Maastrichtian levels $\left(2-3 \mathrm{~g} / \mathrm{cm}_{2} / \mathrm{k}\right.$.y.) until sometime between 64.5 and 
Table 2 (continued).

\begin{tabular}{|c|c|c|c|c|c|c|c|c|c|}
\hline $\mathrm{Ce}$ & Nd & $\mathrm{Sm}$ & Eu & Tb & Dy & $\mathrm{Yb}$ & Lu & $\begin{array}{c}\text { Carb. } \\
(\%)\end{array}$ & $\begin{array}{c}\text { MAR } \\
(\mathrm{g} / \mathrm{cm} 2 \mathrm{ky})\end{array}$ \\
\hline 41.1 & 85.9 & 10.7 & 2.81 & 4.07 & 12.6 & 7.30 & 1.16 & 82.9 & 5.60 \\
\hline 14.4 & 15.8 & 4.90 & 1.21 & 0.356 & 6.89 & $<3.98$ & 0.424 & 69.8 & 5.97 \\
\hline 25.0 & 31.9 & 5.57 & 1.57 & 1.20 & 6.69 & 4.17 & 0.512 & 58.0 & 5.10 \\
\hline 30.9 & 46.2 & 6.19 & 1.36 & 0.936 & 7.52 & 4.08 & 0.552 & 70.1 & 5.86 \\
\hline 29.4 & 16.7 & 5.02 & 1.50 & 2.69 & 5.32 & 3.63 & 0.492 & 39.4 & 4.26 \\
\hline 24.4 & 53.1 & 5.12 & 1.23 & 0.416 & 8.29 & $<3.91$ & 0.424 & 74.8 & 5.45 \\
\hline 32.5 & 38.5 & 7.39 & 1.85 & 1.50 & 9.43 & 4.70 & 0.594 & 78.7 & 1.59 \\
\hline 37.3 & 47.5 & 8.14 & 2.15 & 1.63 & 9.06 & 5.36 & 0.729 & 71.1 & 1.53 \\
\hline 33.0 & 47.0 & 6.53 & 2.16 & 1.23 & 9.76 & 4.28 & 0.582 & 67.8 & 1.51 \\
\hline 41.3 & 44.3 & 8.26 & 2.75 & 1.68 & 9.07 & 5.37 & 0.830 & 76.1 & 1.64 \\
\hline 16.4 & 21.2 & 2.86 & 0.746 & 0.568 & 3.73 & 2.02 & 0.290 & 40.2 & 1.81 \\
\hline 28.5 & 21.8 & 4.70 & 1.34 & 1.03 & 6.39 & 3.32 & 0.431 & 17.8 & 1.07 \\
\hline 35.9 & 23.7 & 5.78 & 1.67 & 1.14 & 7.33 & 4.69 & 0.586 & 2.9 & 1.21 \\
\hline 25.4 & 17.7 & 3.90 & 1.16 & 1.43 & 6.25 & 2.95 & 0.373 & 2.7 & 1.17 \\
\hline 27.7 & 18.2 & 4.29 & 1.28 & 0.877 & 6.52 & 3.40 & 0.444 & 5.9 & 1.18 \\
\hline 26.5 & 18.9 & 4.00 & 1.17 & 0.695 & 5.58 & 3.14 & 0.398 & 7.2 & 1.24 \\
\hline 16.6 & 21.1 & 3.01 & 0.901 & $<0.344$ & 5.28 & 2.79 & 0.366 & 8.5 & 1.22 \\
\hline 32.8 & 12.7 & 4.28 & 1.18 & 0.760 & 5.63 & 3.08 & 0.395 & 10.4 & 1.22 \\
\hline 49.6 & 33.4 & 6.01 & 1.57 & 1.46 & 7.28 & 4.03 & 0.558 & 17.0 & 1.29 \\
\hline 54.0 & 29.9 & 5.71 & 1.01 & 0.746 & 6.29 & 4.14 & 0.599 & 29.2 & 1.33 \\
\hline 35.1 & 18.2 & 3.83 & 0.796 & 0.869 & 4.76 & 2.87 & 0.363 & 27.2 & 1.34 \\
\hline 64.4 & 42.3 & 10.7 & 2.45 & 2.37 & 9.38 & 6.67 & 0.873 & 30.1 & 1.33 \\
\hline 81.3 & 39.8 & 8.77 & 1.31 & 1.63 & 7.45 & 5.56 & 0.637 & 66.3 & 1.82 \\
\hline 26.2 & 26.3 & 5.88 & 1.11 & 1.29 & 7.30 & 4.18 & 0.638 & 42.4 & 1.34 \\
\hline 27.6 & 37.6 & 4.41 & 1.15 & $<0.387$ & 9.05 & 3.56 & 0.459 & 80.8 & 5.67 \\
\hline 40.2 & 69.2 & 8.87 & 1.54 & 1.05 & 8.76 & 6.00 & 0.734 & 72.9 & 4.70 \\
\hline 49.9 & 61.7 & 11.0 & 2.58 & 1.99 & 13.0 & 7.57 & 0.957 & 75.6 & 4.46 \\
\hline 34.0 & 59.1 & 10.4 & 1.98 & 1.90 & 9.06 & 6.28 & 0.836 & 79.4 & 4.52 \\
\hline 43.7 & 42.8 & 9.83 & 1.81 & 1.80 & 12.4 & 6.55 & 0.851 & 79.2 & 4.72 \\
\hline 52.7 & 63.0 & 9.64 & 1.65 & 1.33 & 8.33 & 6.06 & 0.801 & 75.2 & 4.53 \\
\hline 56.6 & 58.9 & 13.7 & 2.89 & 2.49 & 12.1 & 8.77 & 1.23 & 78.1 & 4.92 \\
\hline 47.3 & 43.3 & 13.7 & 2.60 & 1.66 & 12.9 & 9.05 & 1.13 & 78.9 & 4.90 \\
\hline 49.9 & 75.3 & 19.8 & 2.89 & 3.37 & 17.6 & 12.9 & 1.37 & 84.2 & 4.79 \\
\hline 84.3 & 141 & 22.0 & 3.76 & 2.31 & 19.7 & 13.1 & 1.72 & 85.0 & 5.08 \\
\hline 92.8 & 102 & 21.2 & 2.92 & 4.99 & 13.8 & 12.3 & 1.65 & 81.2 & 4.84 \\
\hline 52.9 & 62.3 & 14.3 & 2.78 & 2.29 & 11.3 & 9.22 & 1.03 & 75.6 & 4.85 \\
\hline 57.6 & 71.3 & 12.4 & 2.96 & 3.28 & 13.2 & 9.26 & 1.20 & 81.0 & 5.70 \\
\hline 88.4 & 62.8 & 13.2 & 2.42 & 2.13 & 15.9 & 8.46 & 1.11 & 71.2 & 4.94 \\
\hline 73.8 & 47.6 & 13.4 & 3.52 & 3.66 & 13.2 & 10.4 & 1.35 & 82.2 & 5.48 \\
\hline 73.0 & 45.7 & 9.95 & 2.41 & 2.89 & 7.30 & 8.29 & 1.18 & 81.9 & 5.50 \\
\hline 36.5 & 39.4 & 7.35 & 1.64 & 2.75 & 10.0 & 5.76 & 0.678 & 57.8 & 4.97 \\
\hline 71.4 & 31.9 & 9.50 & 2.10 & 2.21 & 13.6 & 6.85 & 0.962 & 66.4 & 4.99 \\
\hline 164 & 123 & 20.6 & 4.03 & 4.32 & 24.8 & 16.1 & 2.04 & 88.2 & 5.22 \\
\hline 70.4 & 59.8 & 15.1 & 3.96 & 8.20 & 25.9 & 14.1 & 1.93 & 92.0 & 5.35 \\
\hline
\end{tabular}

64.9 $\mathrm{Ma}$; it is not possible to further constrain the timing of this recovery because of the $9 \mathrm{~m}$ gap in core recovery between 336.7 and $345.6 \mathrm{mbsf}$. This suggests that the recovery time for biological productivity following the KT event at Site 752B, then at a paleolatitude of about $50144 \mathrm{~S}$, lasted between 1.5 and $2.0 \mathrm{~m}$.y. In contrast, a significantly faster recovery time $(0.5-1.0 \mathrm{~m} . \mathrm{y}$.) has been noted for some lower latitude Tethyan KT boundary sections (Smit and ten Kate, 1982; Smit and Romein, 1985) and on the Shatsky Rise (Zachos et al., 1989). Whether this result indicates that significant differences exist between the timing of responses to ecological stress of high vs. low latitude biota, or is simply a local phenomenon, awaits further study. It is also possible that the magnitude and duration of the stress applied by the KT event, whatever it was, varied latitudinally.
The relative abundance profile (Fig. 2B) of the volcanogenic component is essentially the mirror image of that of carbonate content (Fig. 4). This is consistent with the fact that biogenic and volcanogenic materials are the two dominant sediment components, and thus should be inversely related when displayed as fractions of whole sediment. Estimates of the flux of biogenic and volcanogenic materials across the KT boundary section are important because they have a direct bearing on the paleoceanographic significance of the $6 \mathrm{~m}$ thick ash sequence which immediately overlies the boundary. Shipboard estimates of sediment flux, based on the visual examination of smear slides, suggest there is a marked decrease in carbonate accumulation (3.94- $0.34 \mathrm{~g} / \mathrm{cm}^{2} / \mathrm{k}$.y.) but relatively little change in ash flux $\left(0.73-0.93 \mathrm{~g} / \mathrm{cm}^{2} / \mathrm{k} . \mathrm{y}\right.$.) across the KT boundary (Rea et al., 1990). 
Table 2 (continued).

\begin{tabular}{|c|c|c|c|c|c|c|c|c|c|c|c|}
\hline $\mathrm{Zn}$ & $C r$ & $\mathrm{Br}$ & Sc & v & $\mathrm{Sb}$ & Co & $\mathrm{Hf}$ & Th & $\mathrm{Se}$ & $\mathrm{Au}$ & La \\
\hline 139 & 71.3 & 57.3 & 20.2 & 197 & $<0.903$ & 17.9 & 2.56 & 1.58 & $<8.24$ & $<0.0327$ & 68.9 \\
\hline 60.6 & 17.2 & 18.0 & 8.71 & 62.0 & $<0.510$ & 10.9 & 1.29 & 0.779 & $<4.74$ & $<0.0175$ & 32.2 \\
\hline 154 & 64.1 & 18.1 & 23.6 & 172 & $<0.483$ & 29.3 & 1.78 & 1.28 & $<4.36$ & 0.0195 & 28.1 \\
\hline 105 & 48.8 & 22.6 & 16.3 & 98.0 & $<0.517$ & 11.8 & 1.63 & 1.33 & $<5.25$ & $<0.0211$ & 35.4 \\
\hline 158 & 82.9 & 17.3 & 28.4 & 213 & 1.73 & 31.4 & 3.15 & 1.32 & $<3.48$ & 0.00925 & 20.8 \\
\hline 69.0 & 44.8 & 29.2 & 11.8 & 61.1 & 2.23 & 9.88 & 1.40 & $<0.436$ & $<5.63$ & $<0.0214$ & 33.2 \\
\hline 138 & 66.2 & 34.0 & 20.4 & 148 & $<0.702$ & 18.2 & 3.93 & 1.85 & $<6.99$ & 0.0235 & 43.3 \\
\hline 199 & 60.1 & 23.7 & 29.8 & 272 & $<0.570$ & 32.7 & 3.49 & 2.76 & $<6.22$ & 0.0280 & 41.8 \\
\hline 157 & 92.0 & 23.6 & 27.9 & 205 & 2.28 & 27.5 & 2.32 & 1.56 & $<5.59$ & 0.0194 & 33.8 \\
\hline 188 & 103 & 28.2 & 31.2 & 207 & 3.39 & 30.9 & 3.22 & 1.57 & $<6.69$ & $<0.0217$ & 48.9 \\
\hline 43.9 & 13.2 & 6.65 & 5.07 & 47.4 & 1.57 & 6.87 & 1.07 & 1.35 & 4.20 & 0.0369 & 18.0 \\
\hline 141 & 95.8 & 19.0 & 27.4 & 202 & 1.33 & 29.3 & 2.31 & 2.28 & 2.91 & 0.0139 & 17.0 \\
\hline 143 & 56.4 & 16.1 & 30.0 & 340 & 1.72 & 46.2 & 3.93 & 1.70 & 5.76 & 0.107 & 14.4 \\
\hline 155 & 66.3 & 18.9 & 28.0 & 332 & 1.19 & 34.3 & 3.23 & 1.31 & 6.43 & $<0.0099$ & 10.3 \\
\hline 162 & 60.9 & 18.5 & 28.7 & 248 & 1.11 & 34.2 & 3.43 & 1.98 & $<2.69$ & $<0.0103$ & 13.2 \\
\hline 150 & 59.3 & 16.4 & 27.4 & 232 & $<0.282$ & 30.3 & 2.99 & 1.60 & 3.42 & 0.115 & 11.7 \\
\hline 129 & 76.7 & 17.7 & 29.5 & 238 & $<0.322$ & 37.5 & 2.44 & 1.25 & 3.73 & 0.0519 & 8.5 \\
\hline 152 & 60.3 & 20.0 & 27.6 & 257 & 1.23 & 27.6 & 2.80 & 1.55 & 2.59 & 0.0218 & 14.2 \\
\hline 519 & 59.4 & 20.4 & 29.9 & 288 & 1.90 & 31.8 & 3.66 & 2.41 & 4.73 & $<0.0116$ & 23.4 \\
\hline 150 & 43.2 & 21.8 & 17.6 & 150 & 1.82 & 27.6 & 9.48 & 11.6 & 6.39 & 0.0218 & 27.8 \\
\hline 99.3 & 68.8 & 21.2 & 19.1 & 150 & 2.03 & 24.1 & 5.69 & 6.23 & 5.21 & 0.0106 & 18.0 \\
\hline 174 & 43.0 & 29.5 & 22.3 & 189 & 0.884 & 19.3 & 5.62 & 6.28 & 5.62 & 0.113 & 40.0 \\
\hline 34.4 & 30.6 & 32.2 & 11.6 & 89.9 & 2.39 & 10.2 & 1.62 & 1.40 & 6.32 & 0.00772 & 61.7 \\
\hline 162 & 79.7 & 28.4 & 26.4 & 234 & $<0.358$ & 29.5 & 2.97 & 1.43 & $<3.75$ & 0.0311 & 29.4 \\
\hline 112 & 27.8 & 34.5 & 6.08 & 90.7 & 3.96 & 15.8 & 1.57 & 1.36 & $<6.86$ & 0.0439 & 39.6 \\
\hline 67.2 & 30.9 & 42.7 & 10.8 & 77.3 & $<0.496$ & 6.86 & 1.68 & 2.24 & $<5.39$ & 0.214 & 58.1 \\
\hline 354 & 102 & 48.4 & 19.2 & 152 & 6.39 & 118 & 4.42 & 5.61 & 16.2 & 0.0332 & 66.6 \\
\hline 113 & 36.9 & 63.0 & 12.7 & 88.0 & $<0.560$ & 6.88 & 2.27 & 2.05 & $<7.09$ & 0.0792 & 68.3 \\
\hline 182 & 29.5 & 57.6 & 13.0 & 92.8 & 3.07 & 12.0 & 3.37 & 3.23 & $<6.91$ & 0.0216 & 67.4 \\
\hline 93.8 & 42.7 & 57.0 & 10.2 & 62.6 & 1.96 & 7.00 & 1.38 & 3.62 & $<6.00$ & 0.0197 & 66.4 \\
\hline 179 & 79.5 & 36.4 & 26.6 & 206 & $<0.806$ & 23.8 & 4.29 & 3.52 & 5.89 & 0.0258 & 86.8 \\
\hline 172 & 117 & 45.0 & 30.9 & 211 & $<0.767$ & 32.1 & 1.74 & 4.58 & $<8.10$ & $<0.0242$ & 86.7 \\
\hline 115 & 58.2 & 92.4 & 21.0 & 122 & 1.79 & 17.7 & 5.82 & 3.03 & $<9.50$ & 0.0209 & 132 \\
\hline 185 & 45.1 & 64.6 & 20.7 & 143 & $<0.863$ & 17.1 & 4.90 & 5.85 & $<9.96$ & $<0.0288$ & 157 \\
\hline 267 & 105 & 58.6 & 21.2 & 188 & 0.413 & 22.0 & 3.73 & 5.08 & $<8.42$ & $<0.0261$ & 151 \\
\hline 175 & 76.3 & 47.6 & 23.7 & 194 & $<0.715$ & 31.1 & 2.97 & 3.33 & $<6.81$ & 0.0299 & 95.1 \\
\hline 78.2 & 56.1 & 37.5 & 19.0 & 141 & 2.60 & 13.4 & 3.88 & 3.39 & $<8.26$ & 0.0942 & 90.5 \\
\hline 215 & 97.1 & 27.8 & 25.0 & 230 & 3.50 & 17.8 & 6.76 & 7.46 & 6.00 & $<0.0215$ & 72.5 \\
\hline 213 & 70.9 & 36.8 & 26.6 & 230 & 3.83 & 26.0 & 4.91 & 5.69 & 16.9 & $<0.0304$ & 91.8 \\
\hline 240 & 56.9 & 33.4 & 27.7 & 229 & 2.29 & 14.3 & 4.00 & $<1.89$ & $<8.95$ & $<0.0282$ & 74.6 \\
\hline 247 & 115 & 19.2 & 31.5 & 273 & 2.75 & 47.4 & 3.18 & 1.95 & 12.1 & $<0.0178$ & 37.5 \\
\hline 164 & 64.6 & 28.9 & 25.0 & 189 & $<0.585$ & 21.3 & 4.23 & 6.79 & $<5.63$ & 0.0750 & 62.8 \\
\hline 170 & 118 & 77.7 & 27.2 & 181 & $<1.23$ & 19.1 & 12.5 & 16.4 & $<13.5$ & 0.0381 & 151 \\
\hline 169 & 101 & 124 & 29.2 & 259 & $<2.05$ & 19.2 & 6.83 & $<1.35$ & $<18.3$ & $<0.0561$ & 140 \\
\hline
\end{tabular}

Our geochemical factor analysis results are in substantial agreement with these flux estimates. As noted above, we report an 11 -fold decrease in accumulation of the biogenic sediment component, which compares well with the 9-fold decrease in carbonate accumulation determined from shipboard studies. Our estimate of ash flux across the KT boundary is more complicated because factor analysis distinguishes between volcanic ash (Factor 2) and diagenetic alteration products (Factor 3). However, even if we assume that all of the material represented by Factor 3 was originally volcanic ash, and thus estimate ash flux as the sum of the MAR's of Factors 2 and 3, (Figs. 3B and 3C), our results also suggest there was little change in ash flux (about 2.5-1.5 $\mathrm{g} / \mathrm{cm}^{2} / \mathrm{k} . \mathrm{y}$.) across the KT boundary. In both cases, then, independent estimates of sediment flux suggest that the ash layer did not result from enhanced volcanic activity, but instead is the result of a dramatic decrease in planktonic productivity immediately following the KT boundary event.
The MAR's determined for Factor 3 typically fall within the range $0.1-1.0 \mathrm{~g} / \mathrm{cm}^{2} / \mathrm{k} . y$., except for two spikes (1.5 and 2.2 $\mathrm{g} / \mathrm{cm}^{2} / \mathrm{k}$.y.) below the KT boundary, both of which correspond to distinct ash layers, and a pronounced spike $\left(3.5 \mathrm{~g} / \mathrm{cm}^{2} / \mathrm{k} . \mathrm{y}\right.$.) at the KT boundary (Fig. 3C). Among all of the elements examined, only $\mathrm{Au}$ is almost exclusively associated with Factor 3 (Table 3). The highest measured Au concentration ( $214 \mathrm{ppb}$ ) was determined for the sample collected just below the KT boundary (358.77 mbsf, Table 1), a finding similar to that reported by Alvarez (1984). No Ir concentrations above the detection limit (3-8 ppb) of the INAA were measured; however, INAA is less sensitive than radiochemical neutron activation analysis, the preferred method for Ir analyses, and there may be $\mathrm{Ir}$ in the lowest boundary clay which we did not sample. Except for Au, all other elements associated with Factor 3 also display strong associations with the biogenic and/or volcanogenic sediment end-members. For example, the non-meteoritic elements $\mathrm{Mn}$ and Se are strongly associated with both the 
Table 3. Age-depth correlation and linear sedimentation rates (LSR) for Hole 752B (from Rea et al., 1990).

\begin{tabular}{ccccc}
\hline $\begin{array}{c}\text { Age } \\
(\mathrm{m} . \mathrm{y} .)\end{array}$ & $\begin{array}{c}\text { Time interval } \\
\text { (m.y.) }\end{array}$ & $\begin{array}{c}\text { Depth } \\
(\mathrm{mbs})\end{array}$ & $\begin{array}{c}\text { Depth interval } \\
(\mathrm{m})\end{array}$ & $\begin{array}{c}\text { LSR } \\
(\mathrm{cm} / \mathbf{k} . \mathbf{y} .)\end{array}$ \\
\hline $63.8-64.8$ & 1.0 & $318.7-345.1$ & 26.4 & 2.64 \\
$64.8-65.9$ & 1.1 & $345.1-353.9$ & 8.8 & 0.80 \\
$65.9-66.4$ & 0.5 & $353.9-358.5$ & 4.6 & 0.92 \\
$66.4-69.0$ & 2.6 & $358.5-422.3$ & 3.85 & 2.4 \\
\hline
\end{tabular}

Table 4. End-member compositions determined by factor analysis (in ppm except where indicated).

\begin{tabular}{|c|c|c|c|}
\hline Element & Factor 1 & Factor 2 & Factor 3 \\
\hline Al $(\%)$ & 4.85 & 11.9 & 11.8 \\
\hline $\mathrm{Fe}(\%)$ & 0.00 & 18.6 & 15.4 \\
\hline$M n(\%)$ & 0.816 & 0.00 & 0.425 \\
\hline $\mathrm{Ba}(\%)$ & 1.60 & 0.470 & 1.24 \\
\hline $\mathrm{Zn}$ & 210 & 431 & 258 \\
\hline Sc & 15.4 & 67.1 & 59.1 \\
\hline $\mathrm{Sb}$ & 5.33 & 2.70 & 0.00 \\
\hline Co & 2.90 & 88.9 & 49.3 \\
\hline Th & 7.80 & 3.96 & 7.45 \\
\hline $\mathrm{Se}$ & 21.0 & 7.12 & 15.2 \\
\hline $\mathrm{Au}$ & 0.0120 & 0.0010 & 0.472 \\
\hline $\mathrm{La}$ & 279 & 0.00 & 181 \\
\hline $\mathrm{Ce}$ & 106 & 192 & 310 \\
\hline Sm & 31.0 & 23.2 & 25.1 \\
\hline Eu & 5.64 & 10.4 & 2.47 \\
\hline $\mathrm{Yb}$ & 22.2 & 20.1 & 18.6 \\
\hline Lu & 2.92 & 2.65 & 2.28 \\
\hline \multicolumn{4}{|l|}{$\%$ of total } \\
\hline variance $=$ & 43.0 & 36.7 & 12.1 \\
\hline \multicolumn{4}{|c|}{ Cummulative variance $=91.8$} \\
\hline
\end{tabular}

biogenic/dissolution residue end-member (Factor 1) and the sediment diagenetic end-member (Factor 3). This association is consistent with the suggestions of Schmitz (1985) and Schmitz et al. (1988) that these elements may become enriched via a mechanism involving redox-controlled and/or microbially mediated precipitation processes. Similarly, high concentrations of $\mathrm{Fe}, \mathrm{Co}, \mathrm{Zn}$, and $\mathrm{Sc}$ are associated with both the volcanic ash (Factor 2) and diagenesis (Factor 3 ) end-members (Table 4). Diagenetic enrichment of certain elements in volcanogenic sediment has been proposed as the origin of the boundary signal by workers who have examined the clay mineralogy and microstratigraphy of other boundary sections (Wezel et al., 1981; Rampino and Reynolds, 1983; Crocket et al., 1988; Elliott et al., 1989). While these elemental associations do not rule out the possibility of contributions from an exotic source, they do suggest that diagenesis of biogenic and volcanogenic sediment may be a viable source for many of the elements anomalously enriched at the KT boundary.

Some authors have suggested that a period of global-scale volcanism may have produced changes in ocean chemistry sufficient to cause the mass extinctions at the KT boundary (Keith, 1982; McLean, 1985; Officer et al., 1987). Our results do not rule out, but certainly do not support this hypothesis. Each of the geochemical end-members identified in this study spans the KT boundary and none is associated exclusively with the sediment above or below the boundary. We infer from this, and from the flux data discussed above, that both the amount and composition of the volcanogenic material deposited at Broken Ridge remained essentially constant before and after the KT boundary event, whereas the composition of the biogenic component remained constant, but the flux was drastically reduced.

\section{SUMMARY}

A Q-mode factor analysis of geochemical data obtained from an expanded section of sediments across the KT boundary at Broken Ridge indicates the sediments here are composed of three major geochemical end-members. The significance of each of these end-members was determined by comparing key inter-element ratios for each end-member with analogous ratios that are considered to be characteristic of specific types of sedimentary material. Based on these comparisons, we have interpreted the three end-members as representing (1) a biogenic/dissolution residue component, (2) a volcanogenic component, and (3) a diagenetic or alteration product component.

We have examined both composition and relative flux of each of these end-members as a means to gain insight into the processes associated with the time represented by the onset, occurrence, and recovery from the KT boundary event at Broken Ridge. For example, the KT boundary section at Broken Ridge is overlain by a 6-m-thick ash-rich layer. A significant question here is whether this layer reflects a sudden increase in ash deposition or, alternatively, a sharp reduction in biological productivity. Our analyses indicate there was an 11-fold decrease in the flux of the biogenic end-member, while the flux of the volcanogenic end-member remained essentially unchanged. This result is in good agreement with preliminary shipboard estimates of sediment flux rates, and suggests the ash layer at Broken Ridge is the result of a dramatic decrease in planktonic productivity immediately following the KT boundary event. The flux of the biogenic component does not return to Maastrichtian levels until 1.5-2.0 m.y. after the KT boundary event, a recovery time that is about $1.0 \mathrm{~m}$.y. longer than that reported for lower latitude KT boundary sections.

The inter-element ratios and REE patterns of the volcanogenic component suggest that the volcanogenic sediments at Broken Ridge are derived from a local source. Both the composition and flux of the volcanogenic component remain constant across the KT boundary, while the composition of the biogenic end-member remains constant but its flux is significantly reduced. Consequently, our results do not indicate that volcanism played a critical role in the KT boundary event.

There is a sharp increase in the flux of the diagenetic end-member coincident with the KT boundary. This end-member contains enriched amounts of $\mathrm{Au}$, and the highest $\mathrm{Au}$ concentration occurs in a sample recovered from just below the KT boundary. Other workers have also reported Au enrichments in sediments from immediately below the KT boundary (Crocket and Kao, 1979), but no agreement exists concerning whether these enrichments represent evidence for the impact hypothesis, or if they are simply the result of diagenetic processes. Except for Au, all other elements that are enriched at the KT boundary are also strongly associated with either or both the biogenic and/or the volcanogenic end-members. We believe the most plausible explanation for geochemical anomalies in the sediments immediately below the KT boundary at Broken Ridge is that they occur as a result of enhanced diagenetic processing which serves to concentrate certain elements originally present in biogenic and volcanogenic sediment precursors.

\section{ACKNOWLEDGMENTS}

We thank our colleagues on board the Resolution during ODP Leg 121 for their assistance in obtaining the samples and gener- 
Table 5. Inter-elemental ratios of Factor 2, Factor 3, and literature values for volcanogenic sediment.

\begin{tabular}{|c|c|c|c|c|c|}
\hline Element & Factor 2 & Factor 3 & $\begin{array}{c}\text { aSantonian } \\
\text { Ash }\end{array}$ & $\begin{array}{c}\text { bKerguelen } \\
\text { Basalt }\end{array}$ & $\begin{array}{l}\text { c Site } 253 \\
\text { Volc. Sed. }\end{array}$ \\
\hline $\mathrm{Al} \% / \mathrm{Fe} \%$ & 0.637 & 0.767 & 0.619 & $0.76 / 0.95$ & 0.796 \\
\hline $\mathrm{Mn} \% / \mathrm{Fe} \%$ & 0.00 & 0.0279 & 0.0140 & $0.030 / 0.033$ & 0.010 \\
\hline Ba ppm/Fe\% & 252 & 806 & 109 & $122 / 45$ & 15.6 \\
\hline $\mathrm{Zn} \mathrm{ppm/Fe \%}$ & 23.1 & 16.7 & 17.5 & - & - \\
\hline $\mathrm{Sc} p \mathrm{ppm} / \mathrm{Fe}_{\mathrm{O}} \%$ & 3.60 & 3.83 & 3.83 & $6.18 / 8.37$ & - \\
\hline $\mathrm{Sb} \mathrm{ppm} / \mathrm{Fe} \%$ & 0.145 & 0.00 & 0.251 & - & - \\
\hline Co ppm/Fe\% & 4.77 & 3.19 & 3.83 & - & 6.60 \\
\hline Th ppm/Fe\% & 0.213 & 0.483 & 0.190 & $0.94 / 0.38$ & - \\
\hline Co ppm/Al\% & 7.50 & 0.600 & 6.19 & - & 8.26 \\
\hline Zn ppm/Al\% $\%$ & 36.0 & 43.0 & 26.9 & - & 17.5 \\
\hline LaYrb & - & 9.70 & 5.06 & $24.0 / 7.95$ & 4.46 \\
\hline $\mathrm{Sm} / \mathrm{Lu}$ & 8.68 & 11.0 & 9.62 & $22.9 / 12.5$ & 9.48 \\
\hline $\mathrm{Sm} / \mathrm{Eu}$ & 2.21 & 10.0 & 3.31 & $3.69 / 2.91$ & 3.71 \\
\hline SmYb & 1.14 & 1.34 & 1.30 & $3.29 / 1.81$ & 1.42 \\
\hline
\end{tabular}

a Our mean analyses of a Santonian ash layers in ODP Sections 121-755A-5-1 to 121-755A-9-2.

bKerguelen alkali basalttholeiite data from Storey et al. (1988).

c Middle Eocene volcanogenic sediment sequence DSDP Site 253, mean of Units I-VI. REE's are from Fleet et al. (1976). All other elements from Fleet and McKelvey (1978).

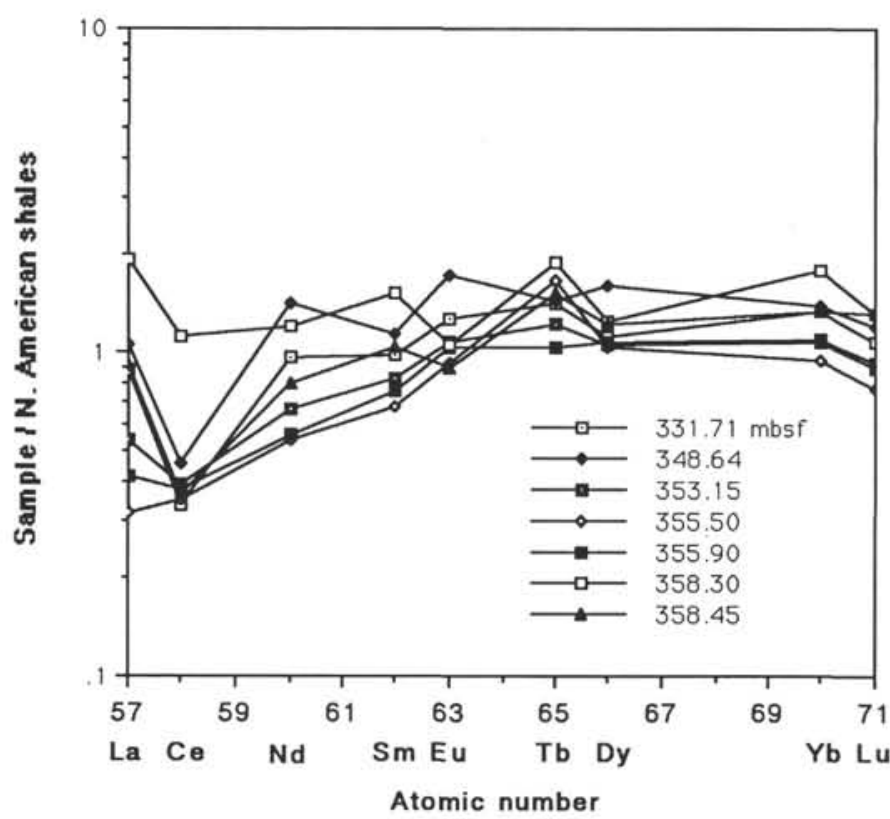

Figure 1. Typical REE patterns of KT section samples. All data on a carbonate-free basis and normalized to a North American shale composite (Gromet et al., 1984).

ating the shipboard data used in this study, and for making a long cruise a very pleasant one. M. Lyle, J. Weissel, and an anonymous reviewer provided helpful comments and suggestions based on a review of an earlier version of this paper. This research was supported by Contract No. 20252 from the Texas A\&M Research Foundation awarded to R. M. Owen.

\section{REFERENCES}

Alvarez, L. W., Alvarez, W., Asaro, F., and Michel, H. V., 1980. Extraterrestrial cause for the Cretaceous-Tertiary extinction. Science, 208:1095-1108.

Alvarez, W., 1984. The end of the Cretaceous: sharp boundary or gradual transition? Science, 223:1183-1186.

Alvarez, W., Alvarez, L. W., Asaro, F., and Michel, H. V., 1982. Current status of the impact theory for the terminal Cretaceous extinction. In Silver, L. T., and Schultz, P. H. (Eds.), Geological Implications of Impacts of Large Asteroids and Comets on the Earth. Spec. Pap. Geol. Soc. Am., 190:305-315.

Arthur, M. A., Zachos, J. C., and Jones, D. S., 1987. Primary productivity and the Cretaceous/Tertiary boundary event in the oceans. Cretaceous Res., 8:43-54.

Berggren, W. A., Kent, D. V., Flynn, J. J., and Van Couvering, J. A., 1985. Cenozoic geochronology. Geol. Soc. Am. Bull., 96:1407-1418.

Boström, K., Joensuu, D., Moore, C., Boström, B., Dalziel, M,, and Horowitz, A., 1973. Geochemistry of barium in pelagic sediments. Lithos, 6:159-174.

Chester, R., and Aston, S. R., 1976. The geochemistry of deep sea sediments. In Riley, J. P., and Chester, R. (Eds.), Chemical Oceanography (2nd ed.) (Vol. 6): New York (Academic Press), 281-390.

Crocket, J. H., and Kuo, H. Y., 1979. Sources for gold, palladium and iridium in deep-sea sediments. Geochim. Cosmochim. Acta, 43:831842.

Crocket, J. H., Officer, C. B., Wezel, F. C., and Johnson, G. D., 1988. Distribution of noble metals across the Cretaceous/Tertiary boundary at Gubbio, Italy: iridium variations as a constraint on the duration and nature of Cretaceous/Tertiary boundary events. Geology, 16:77-88.

Dams, R., and Robbins, J. A., 1970. Nondestructive activation analysis of environmental samples. Univ. Mich. Great Lakes Res. Div. Tech. Rept., 48:1-92.

Dehairs, R., Chesselet, R., and Jedwab, J., 1980. Discrete suspended particles of barite and the barium cycle in the open ocean. Earth Planet. Sci. Lett., 49:528-550.

DePaolo, D. J., Kyte, F. T., Marshall B. D., O'Neil, J. R., and Smit, J., 1983. Rb-Sr, Sm-Nd, K-Ca, 0, and hisotopic study of Cretaceous-Tertiary boundary sediments, Caravaca Spain: evidence for an oceanic impact site. Earth Planet. Sci. Lett., 64:356-373. 
Despraires, A., and Bonnot-Courtois, C., 1980. Relation entre la composition des smectites d'alteration sous-marine et leur cortege de terres rares. Earth Planet. Sci. Lett., 48:124-130.

Dyer, B. D., Lyalikova, N. N., Murray, D., Doyle, M., Kolesov, G. M., and Krumbein, W. E., 1989. Role of microorganisms in the formation of iridium anomalies. Geology, 17:1036-1039.

Dymond, J., 1981. The geochemistry of Nazca Plate sediments: an evaluation of hydrothermal, biogenic, detrital, and hydrogenous sources. In Kulm, L. D., Dymond, J., Dasch, D. M., and Hussong, D. M. (Eds.), Nazca Plate: Crustal Formation and Andean Convergence. Geol. Soc. Am. Mem., 154:133-174.

Elderfield, H., Hawkesworth, C. J., and Greaves, M. G., 1981. Rare earth element geochemistry of oceanic ferromanganese nodules and associated sediment. Geochim. Cosmochim. Acta, 45:513-528.

Elliott, W. C., Aronson, J. L., Millard, Jr., H. T., and Gierlowski-Kordesch, E., 1989. The origin of the clay minerals at the Cretaceous/Tertiary boundary in Denmark. Geol. Soc. Am. Bull., 101:702-710.

Fischer, A. G., and Arthur, M. A., 1977. Secular variations in the pelagic realm. Spec. Publ. Soc. Econ. Paleontol. Mineral., 25:19-50.

Fleet, A. J., 1984. Aqueous and sedimentary geochemistry of the rare earth elements. In Henderson, P. (Ed.), Rare Earth Element Geochemistry. Developments in Geochem., 2:343-373.

Fleet, A. J., Henderson, P., and Kempe, D.R.C., 1976. Rare earth element and related chemistry of some drilled southern Indian Ocean basalts and volcanogenic sediments. J. Geophys. Res., 81:4257-4268.

Fleet, A. J., and McKelvey, B. C., 1978. Eocene explosive submarine volcanism, Ninetyeast Ridge, Indian Ocean. Mar. Geol., 26:73-97.

Ganapathy, R., 1980. A major meteorite impact on the earth 65 million years ago: evidence from the Cretaceous-Tertiary boundary clay. Science, 209:921-923.

Gilmore, J. S., Knight, J. D., Orth, C. J., Pilmore, C. L., and Tschudy, R. H., 1984. Trace element patterns at a non-marine Cretaceous-Tertiary boundary. Nature, 307:224-228.

Gladney, E. S., O'Malley, B. T., Roelandt, I., and Gills, T. E., 1987. Standard reference materials: compilation of elemental concentration data for NBS clinical, biological geological, and environmental standard reference materials. NBS Spec. Publ., 260-111.

Goldschmidt, V. M., 1954. Geochemistry: Oxford (Oxford Univ. Press).

Gordon, C. E., Randle, K., Goles, G. D., Corliss, J. B., Beesen, M. H., and Oxley, S. S., 1968. Instrumental analysis of standard rocks with high resolution X-ray detectors. Geochim. Cosmochim. Acta, 32: 364-396.

Graybeal, A. L., and Heath, G. R., 1984. Remobilization of transition metals in surficial pelagic sediments from the Eastern Pacific. Geochim. Cosmochim. Acta, 48:965-975.

Gromet, L. P., Dymek, R. F., Haskin, L. A., and Korotev, R. L., 1984. The "North American Shale Composite": its compilation, major and trace element characteristics. Geochim. Cosmochim. Acta, 48:24692482.

Gurvich, Y. G., Bogdanov, Y. A., and Lisitsin, A. P., 1978. Behavior of barium in recent sedimentation in the Pacific: Trans. from Geokhimiya (Scripta Publ. Co.), 3:359-374.

Harland, W. B., Cox, A. V., Llewellyn, P. G., Pickton, C.A.G., Smith, D. G., and Walters, R., 1982. A Geologic Time Scale: Cambridge (Cambridge Univ. Press).

Kastner, M., Asaro, F., Michel, H. V., Alvarez, W., and Alvarez, L. W., 1984. The precursor of the Cretaceous-Tertiary boundary clays at Stevns Klint, Denmark, and DSDP Hole 465A. Science, 226:137-143.

Keith, M. L., 1982. Violent volcanism, stagnant oceans and some inferences regarding petroleum, strata-bound ores and mass extinctions. Geochim. Cosmochim. Acta, 46:2621-2637.

Kent, D. V., and Gradstein, F. M., 1985. A Cretaceous and Jurassic geochronology. Geol. Soc. Am. Bull., 96:1419-1427.

Kyte, F. T., and Wasson, J. T., 1982. Geochemical constraints on the nature of large accretionary events. Spec. Pap. Geol. Soc. Am., 190:235-242.

Kyte, F. T., Zhou, Z., and Wasson, J. T., 1980. Siderophile-enriched sediments from the Cretaceous-Tertiary boundary. Nature, 288:651656.
Leinen, M., and Pisias, N., 1984. An objective technique for determining end-member compositions and for partitioning sediments according to their sources. Geochim. Cosmochim. Acta, 48:47-62.

Ludden, J. N., and Thompson, G., 1978. Behavior of rare earth elements during submarine weathering of tholeiitic basalt. Nature, 274:147149.

Marchig, V., Gundlach, H., Moller, P., and Schley, F., 1982. Some geochemical indicators for discrimination between diagenetic and hydrothermal metalliferous sediments. Mar. Geol., 50:241-256.

Martin, J. H., and Knauer, G. A., 1973. The elemental composition of plankton. Geochim. Cosmochim. Acta, 37:1639-1653.

Mayer, L. M., Macko, S. A., Mook, W. H., and Murray, S., 1981. The distribution of bromine in coastal sediments and its use as a source indicator for organic matter. Org. Geochem., 3:37-42.

McLean, D. M., 1985. Deccan Traps mantle degassing in the terminal Cretaceous marine extinctions. Cretaceous Res., 6:235-259.

Officer, C. B., and Drake, C. L., 1983. The Cretaceous-Tertiary transition. Science, 219:1383-1390.

Officer, C. B., Hallam, A., Drake, C. L., and Devine, J. D., 1987. Late Cretaceous and paroxysmal Cretaceous/Tertiary extinctions. Nature, 326:143-149.

Okada, H., and Bukry, D., 1980. Supplementary modification and introduction of code numbers to the low latitude coccolith biostratigraphic zonation (Bukry, 1973; 1975). Mar. Micropaleontol., 5:321-325.

Olmez, I., Finnegan, D. L., and Zoller, W. H., 1986. Iridium emissions from Kilauea volcano. J. Geophys. Res., 91:653-663.

Owen, R. M., and Ruhlin, D. E., 1986. Interlaboratory comparison of Leg 92 standard sediment sample analyses. In Leinen, M., Rea, D. K. et al., Init. Repts. DSDP, 92: Washington (U.S. Govt. Printing Office), 353-354.

Palmer, M. R., 1985. Rare earth elements in foraminifera test. Earth Planet. Sci. Lett., 73:285-298.

Peirce, J., Weissel, J., et al., 1989. Proc. ODP, Init. Repts., 121: College Station, TX (Ocean Drilling Program)

Piper, D. Z., 1974a. Rare earth elements in the sedimentary cycle: a summary. Chem. Geol., 14:285-304.

1974b. Rare earth elements in ferromanganese nodules and other marine phases. Geochim. Cosmochim. Acta, 38:1007-1022.

Price, N. B., and Calvert, S. E., 1977. The contrasting geochemical behaviors of iodine and bromine in recent sediments from the Namibian shelf. Geochim. Cosmochim. Acta, 41:1769-1775.

Rampino, M. R., and Reynolds, R. C., 1983. Clay mineralogy of the Cretaceous-Tertiary boundary clay. Science, 219:495-498.

Rea, D. K., Dehn, J., Driscoll, N., Farrell, J., Janecek, T., Owen, R. M., Pospichal, J. L., Resiwati, P., and the ODP Leg 121 Scientific Party, 1990. Paleoceanography of the Eastern Indian Ocean from ODP Leg 121 Drilling on Broken Ridge. Geol. Soc. Am. Bull., 102:679-690.

Reddy, V. V., Subbaroa, K. V., Reddy, G. R., Matsuda, J., and Hekinian, R., 1978. Geochemistry of volcanics from the Ninetyeast Ridge and its vicinity in the Indian Ocean. Mar. Geol., 26:99-117.

Riley, J. P., and Roth, I., 1971. The distribution of trace elements in some species of phytoplankton grown in culture. J. Mar. Biol. Assoc. U.K., 51:63-72.

Ruhlin, D. E., and Owen, R. M., 1986. The rare earth element geochemistry of hydrothermal sediments from the East Pacific Rise: examination of a seawater scavenging mechanism. Geochim. Cosmochim. Acta, 50:393-400.

Schmitz, B., 1985. Metal precipitation in the Cretaceous- Tertiary boundary clay at Stevns Klint, Denmark. Geochim. Cosmochim. Acta, 49:2361-2370.

1987. Barium, equatorial high productivity, and the northward wandering of the Indian continent. Paleoceanography, 2:63-77.

Schmitz, B., Andersson, P., and Dahl, J., 1988. Iridium, sulfur isotopes and rare earth elements in the Cretaceous-Tertiary boundary clay at Stevns Klint, Denmark. Geochim. Cosmochim. Acta, 52:229-236.

Shimizu, H., and Masuda, A., 1977. Cerium in chert as an indication of marine environment of its formation. Nature, 266:346-348.

Sissingh, W., 1977. Biostratigraphy of Cretaceous calcareous nannoplankton. Geol. Mijnbouw, 56:37-65. 
Smit, J., and Romein, A.J.T., 1985. A sequence of events across the Cretaceous-Tertiary boundary. Earth Planet. Sci. Lett., 74:155-170.

Smit, J., and ten Kate, W.G.H.Z., 1982. Trace element patterns at the Cretaceous-Tertiary boundary - consequences of a large impact. Cretaceous Res., 3:307-332.

Storey, M., Saunders, A. D., Tarney, J., Leat, P., Thirlwall, M. F., Thompson, R. N., Menzies, M. A., and Marriner, G. F., 1988. Geochemical evidence for plume-mantle interactions beneath Kerguelen and Heard Islands, Indian Ocean. Nature, 336:371-374.

Tappan, H., 1968. Primary production, isotopes, extinctions and the atmosphere. Palaeogeogr., Palaeoclimatol., Palaeoecol., 4:185-210.

Thierstein, H. R., 1981. Late Cretaceous nannoplankton and the change at the Cretaceous-Tertiary boundary. In Warme, J. E., et al. (Eds.), The Deep Sea Drilling Project: A Decade of Progress: Soc. Econ. Paleontol. Mineral., Spec. Publ., 32:355-394.

Wezel, F. C., Vannucci, S., and Vannucci, R., 1981. Decouverte de divers niveaux riches en iridium dans la "Scaglia rossa" et al "Scaglia bianca" de 1'Alpennin d'Ombrie-Marches (Italie), C.R. Acad. Sci. Paris Ser. 2, 293:837-844.

Zachos, J. C., and Arthur, M. A., 1986. Paleoceanography of the Cretaceous/Tertiary boundary event: inferences from stable isotopic and other data. Paleoceanography, 1:5-26.

Zachos, J. C., Arthur, M. A., and Dean, W. E., 1989. Geochemical evidence for suppression of pelagic marine productivity at the Cretaceous/Tertiary boundary. Nature, 337:61-64.

Zoller, W. H., Parrington, J. R., and Kotra, J.M.P., 1983. Iridium enrichment in airborne particles from Kilauea volcano: January 1983. Science, 222:1118-1121.

Date of initial receipt: 14 December 1989

Date of acceptance: 14 August 1990

Ms 121B-147
A.

Factor 1

(\% composition)

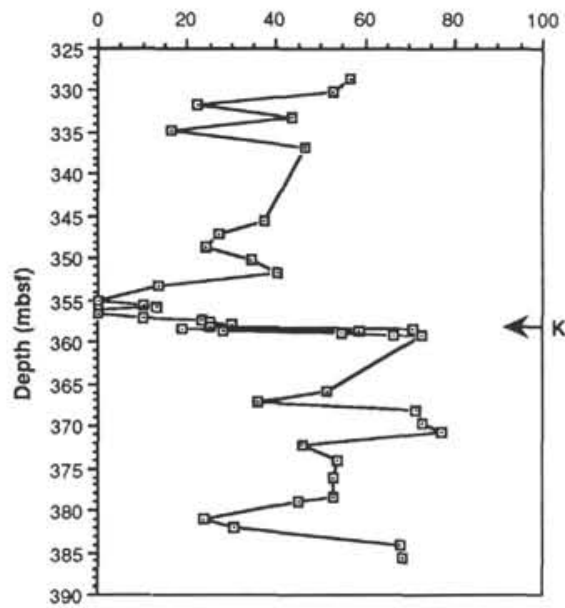

B.

Factor 2

(\% composition)

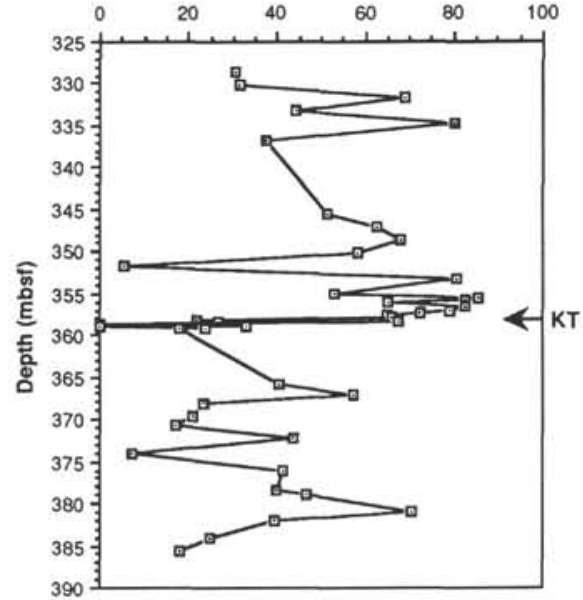

C.

Factor 3

Figure 2. A. Downcore relative contribution of Factor 1 (biogenic) sediment. B. Factor 2 (volcanogenic). C. Factor 3 (diagenetic). Placement of the KT boundary is as suggested by Peirce, Weissel, et al. (1989).

A.

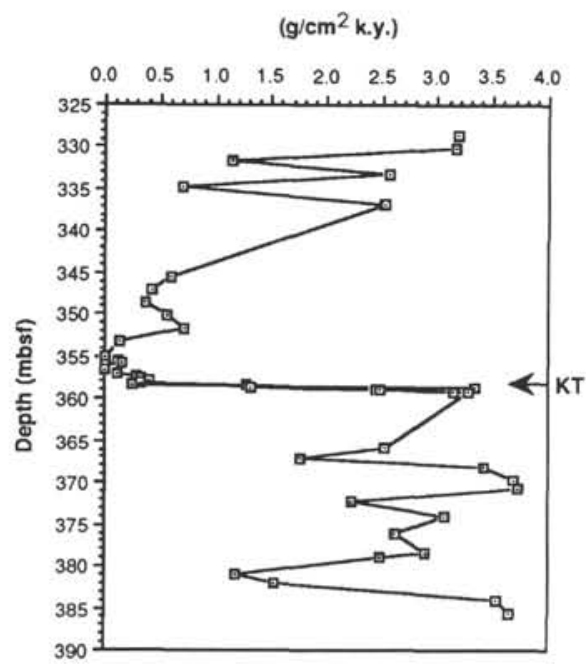

B.

Factor 2 - MAR

$\left(g / \mathrm{cm}^{2}\right.$ k.y.)

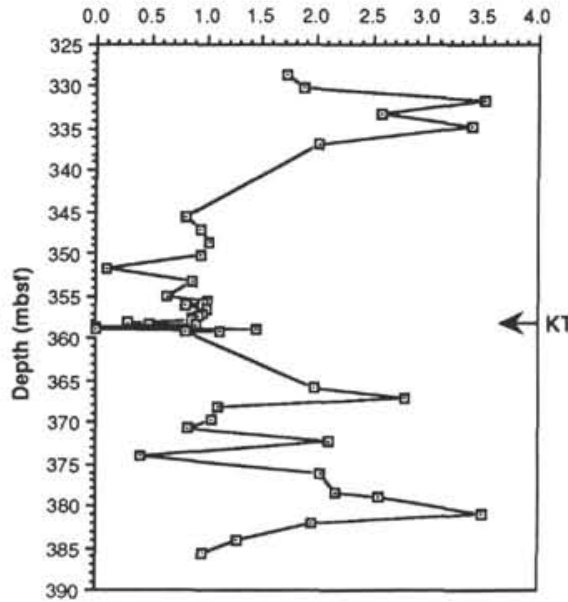

c.

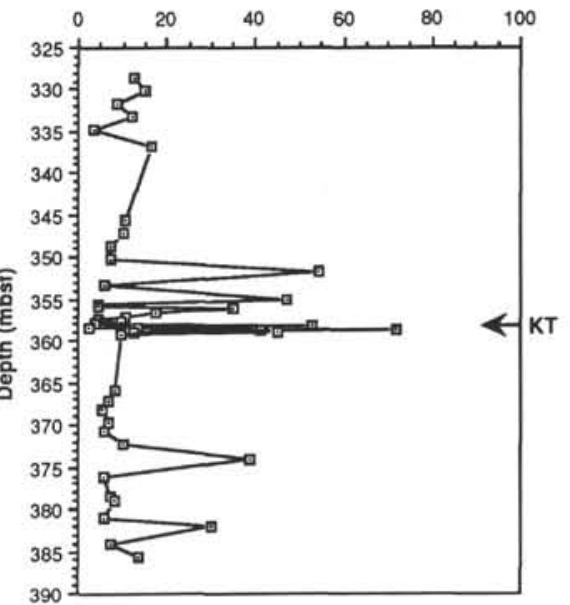

Figure 3. A. Downcore mass accumulation rate of Factor 1 (biogenic) sediment. B. Factor 2 (volcanogenic). C. Factor 3 (diagenetic). Factor MAR's were calculated as the product of whole sediment mass accumulation rate (for each sample) and the relative contribution of each factor (to each sample). 
Carbonate content (\%)

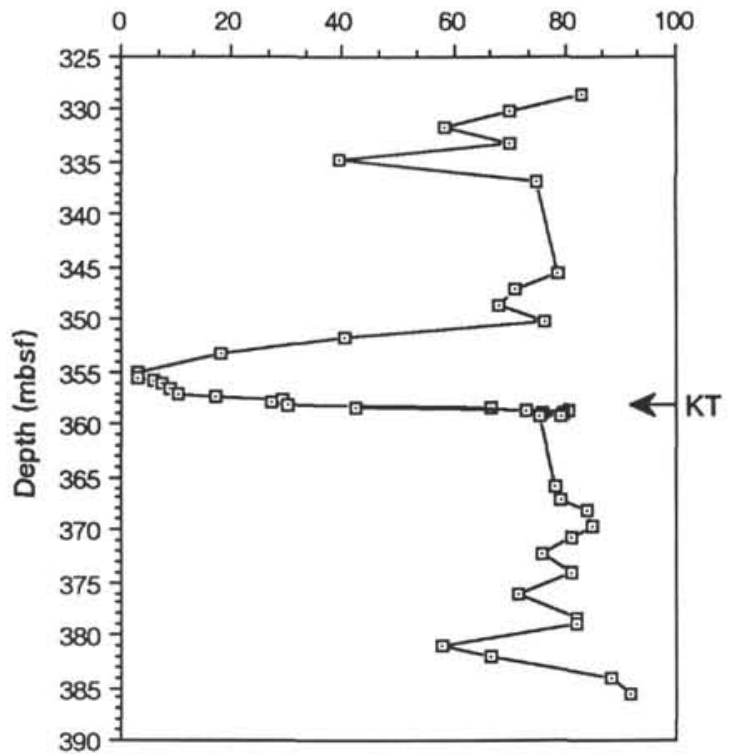

Figure 4. Downcore carbonate content determined by carbonate bomb technique. 